\title{
Reactors antineutrino anomalies and searches for sterile neutrinos in Europe
}

\author{
Michel Cribier ${ }^{1}$ \\ Affiliation \\ Address, Country \\ E-mail: michel.cribier@cea.fr
}

Over the last 20 years a standard neutrino oscillation framework associated with small splitting between the neutrino mass states have become well established. Beyond this model, anomalies have been observed at short baseline in reactor, accelerator and gallium experiments. This suggests the existence of a fourth massive neutrino, affecting experiments through oscillation with active flavours with $\Delta \mathrm{m}^{2}$ above $0.1 \mathrm{eV}^{2}$. To definitively test this $\Delta \mathrm{m}^{2}$ region, several experiments on reactors or using neutrino or antineutrino sources are in preparation.

XV Workshop on Neutrino Telescopes

March 11-15, 2013

Venice, Italy

1

Speaker 


\section{Introduction}

Most results from neutrino experiments over the last 20 years can be quite accurately described by a model of oscillations between three $v$ flavors $\left(v_{e}, v_{\mu}, v_{r}\right)$ that are mixtures of three massive neutrinos $\left(v_{l}, v_{2}, v_{3}\right)$ separated by squared mass differences of $\Delta m_{21}^{2}=8 \times 10^{-5} \mathrm{eV}^{2}$ and $\Delta m_{31}^{2}=2.4 \times 10^{-3} \mathrm{eV}^{2}$. Apart from the confusing results of the LSND and MiniBoone experiments the hypothetical existence of a fourth $v$ has been revived by a new calculation of the rate of $\bar{v}_{e}$ production by nuclear reactors [1] that yields a $\bar{v}_{e}$ flux about $3 \%$ higher than previously predicted. This calculation then implies [2] that the measured event rates for all reactor $\bar{v}_{e}$ experiments within 100 meters of the reactor are about $6 \%$ too low. The deficit can also be explained by a hypothetical fourth massive $v$ separated from the three others by a new $\Delta \mathrm{m}^{2}>0.1 \mathrm{eV}^{2}$. This mixing can explain a similar deficit in the rate of $v$ interactions in gallium solar- $v$ detectors when exposed to artificial ${ }^{51} \mathrm{Cr}$ and ${ }^{37} \mathrm{Ar} \mathrm{MCi}$ sources. The combination of these deficits is significant at the $99.8 \%$ C.L. In this contribution we will present some of the several experiments under construction in Europe to test this hypothesis.

\section{Experimental evidences pointing toward a fourth neutrino}

Several sets of experimental results could be explained by the presence of a fourth neutrino. In particular experiments of antineutrinos at moderate distance $(<100 \mathrm{~m})$ of nuclear reactors on one hand and results of calibration of gallium solar neutrinos experiments with neutrino sources on the other hand point to oscillation toward a fourth neutrino state with very similar parameters of oscillation. Although every single results is not conclusive by itself, the whole set of results is coherent and thus call for experimental verification.

\subsection{Reactor antineutrino anomaly}

Nuclear reactors are very intense sources of neutrinos that have been used all along the neutrino's history, from its discovery up to the most recent oscillation studies. With an average energy of about $200 \mathrm{MeV}$ released per fission and 6 neutrinos produced along the B-decay chains of the fission products, one expects about $2 \times 10^{20} \mathrm{v} / \mathrm{s}$ emitted in a $4 \pi$ solid angle from a 1 GW reactor (thermal power). Since unstable fission products are neutron-rich nuclei, all $\beta-$ decays are of $\beta^{-}$type and the neutrino flux is actually pure electronic antineutrinos $\left(\bar{v}_{e}\right)$. The neutrino oscillation searches at a reactor is always based on a disappearance measurement, using the powerful inverse beta decay (IBD) detection process to discriminate the neutrino signal from backgrounds. The observed neutrino spectrum at a distance $\mathrm{L}$ from a reactor is compared to the expected spectrum. If a deficit is measured it can be interpreted in terms of the disappearance probability which, in the two neutrino mixing approximation, reduces to :

$$
P_{e e}=1-\sin ^{2}(2 \theta) \times \sin ^{2}\left(\frac{\Delta m^{2} L}{2 E}\right)
$$

with $\Delta \mathrm{m}^{2}$ the difference between the squared masses of the two neutrino states and $\theta$ the mixing angle fixing the amplitude of the oscillation. In the last 20 years reactor antineutrino 
experiments were performed at distances below $100 \mathrm{~m}$ from the reactor core, in particular ILLGrenoble, Goesgen, Rovno, Krasnoyarsk, Savannah River and Bugey. These experiments employed only one detector and therefore depend on an accurate theoretical prediction for the emitted $\bar{v}_{e}$ flux and spectrum to measure $P_{e e}$. Until late 2010, all data from reactor neutrino experiments measured a rate of $\bar{v}_{e}$ in reasonable agreement with that predicted from the 'old' reactor antineutrino spectra [3], though slightly lower than expected, with the measured/expected ratio $\mathrm{R}$ at $0.980 \pm 0.024$, where the recent neutron mean lifetime, of $881.5 \mathrm{~s}$ is used to compute the cross section of the detection reaction of $\bar{v}_{e}$ on free protons :

$$
\bar{v}_{e}+p \rightarrow n+e^{+}
$$

\subsubsection{Reevaluation of reactor antineutrino experiments}

In preparation for the Double Chooz reactor experiment, the Saclay reactor neutrino group re-examined the specific reactor antineutrino flux for ${ }^{235} \mathrm{U},{ }^{239} \mathrm{Pu},{ }^{241} \mathrm{Pu}$, and ${ }^{238} \mathrm{U}$ using the new existing nuclear data. In 2011, they reported their results [1], which correspond to a flux that is a few percent higher than the previous prediction. Based on this new antineutrino fluxes a reanalysis of the ratio of observed event rate to predicted rate for 19 published experiments at reactor-detector distances below $100 \mathrm{~m}$ was performed.

In summary, this re-evaluation has revealed some systematic biases in the previously published conversion of the ILL electron data. The net result is a $+3 \%$ shift in the predicted emitted spectra. The origin of these biases were not in the principle of the conversion method but in the approximate treatment of nuclear data and corrections to the Fermi theory. A complementary work [4] confirmed the origin of the biases and showed that an extra correction term should be added increasing further the predicted antineutrino spectra at high energy. These most recent spectra are the new reference used for the analysis of the reactor anomaly in the next section. The prediction of the last isotope contributing to the neutrino flux of reactors, ${ }^{238} \mathrm{U}$, is also updated by $a b$ initio calculations.

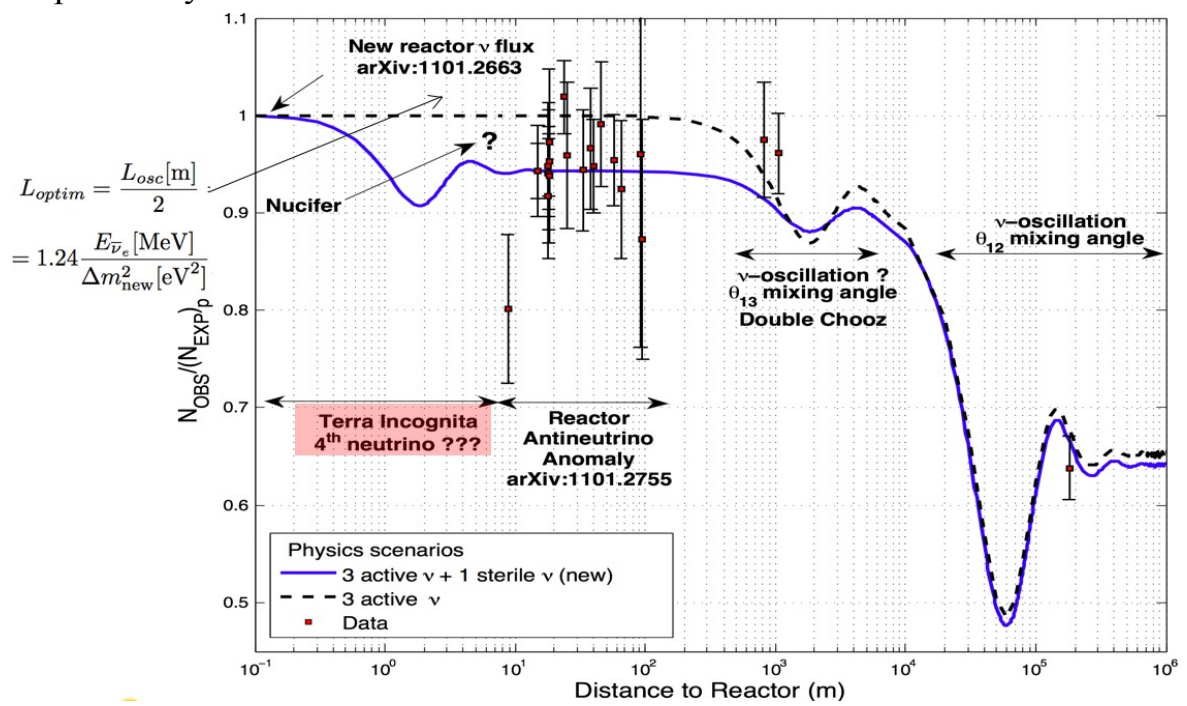

Figure 1. Illustration of the short baseline reactor antineutrino anomaly

With this re-analysis of these experiment the ratio for all these reactor experiments measured/expected $\mathrm{R}=0.927 \pm 0.023$. Moreover the first ILL experiment performed in 1980 at 
less than $9 \mathrm{~m}$ from the core and reanalysed in 1995 [5] show an even greater deficit and even an intriguing structure in the energy spectrum.

\subsection{Gallium neutrino anomalies}

The GALLEX and SAGE solar neutrino detectors have been tested in so-called "Gallium radioactive source experiments" which consist in the detection of electron neutrinos produced by intense artificial ${ }^{51} \mathrm{Cr}$ and ${ }^{37} \mathrm{Ar}$ radioactive sources placed inside the detectors. These radioactive nuclei decays through electron capture, emitting $v_{e}$ lines at energies below $1 \mathrm{MeV}$.

The neutrinos emitted by the radioactive sources have been detected through the same reaction used for the detection of solar neutrinos [431] $v_{e}+{ }^{71} G a \rightarrow{ }^{71} G e+e^{-}$. In total four source experiments have been performed. The average ratios of measured to predicted ${ }^{71} \mathrm{Ge}$ production rates in the GALLEX and SAGE source experiments is $\mathrm{R}=0.86 \pm 0: 05$. Thus, the number of measured events is about $2.8 \sigma$ smaller than the prediction. This is the "Gallium anomaly", which could be also a manifestation of short-baseline neutrino oscillations.

\subsection{Global fits}

Each set of experiments fitted separately leads to similar values of $\sin ^{2}\left(2 \theta_{\text {new }}\right)$ and similar lower bounds for $\Delta m_{n e w}^{2}$. Hence, we performed a global fit of these results [2] taking into account the existing correlations between experimental results. This leads to a solution for a new neutrino oscillation, such that $\Delta m_{\text {new }}^{2}>1.5 \mathrm{eV}^{2}(95 \%$ C.L. $)$ and $\sin ^{2}\left(2 \theta_{\text {new }}\right)=0.14 \pm 0.08$ (95\% C.L.), disfavoring the no-oscillation case at 99.8\% C.L [2] illustrated in fig. 2.

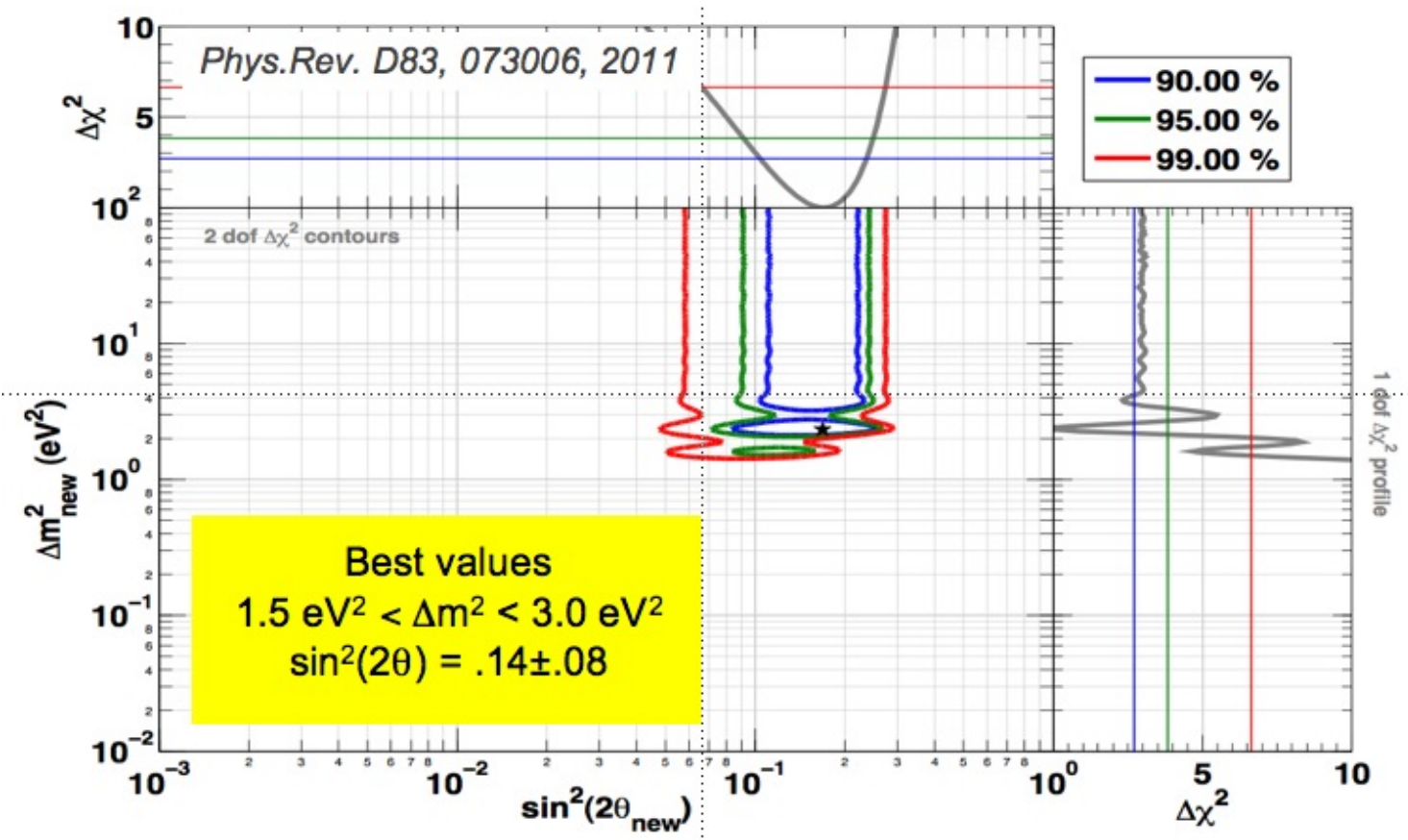

Figure 2. Allowed regions in the parameter space from the combination of reactor neutrino experiments, Gallex and Sage calibration sources experiments, 


\section{Experiment tests of a fourth neutrino}

Clearly the possible existence of a fourth neutrino suggested by the above results call for experimental tests. In that respect several groups around the world are preparing experiments using different techniques. Apart from experiments using beams of neutrinos discussed in this issue, these efforts can be divided in experiments at very close distance of a nuclear reactor and experiments using man-made neutrino and antineutrinos sources.

\subsection{Experimental projects on reactors}

Several projects on reactors in Europe are planned and I had to choose, by lack of space, only three of them. I refer to reference [6] for a wider selection, but I just want to mention the Solio detector in preparation with a ${ }^{6} \mathrm{Li}$ detector.

\subsubsection{Nucifer}

The Nucifer [7] detector target is a stainless steel vessel of $1.8 \mathrm{~m}$ in height, and $1.2 \mathrm{~m}$ in diameter filled with about $0.85 \mathrm{~m}^{3}$ of Gd-doped liquid scintillator (EJ335 from Eljen technology). The internal surface of the vessel is coated with Teflon to ensure the compatibility with the liquid scintillator and to increase the light reflections. All mechanical parts, in particular welding materials, are low radioactive materials. The photodetection system is based on 16 large (8 inches in diameter, R5912) photomultipliers (PMTs) from Hamamatsu, providing a large dynamic of light detection from the single photoelectron to few hundreds of photoelectrons and ensuring an efficient light collection. PMTs are coupled to a $250 \mathrm{~mm}$ thick acrylic vessel placed at the top of the target vessel. This so-called acrylic buffer aims at ensuring the uniformity of the response in the whole target volume while reducing the light generated by the intrinsic PMT radioactivity in the scintillator. 80 liters of mineral oil are used to ensure the optical coupling between the PMTs and the acrylic.

The Nucifer experiment take data at the Osiris research reactor $\left(70 \mathrm{MW}_{\text {th }}\right)$ at only 7 meters from the core. This unique configuration, short distance and the compactness of Osiris core reduces the dispersion of neutrino paths. If an $\mathrm{eV}^{2}$ sterile neutrino exists the measured energy spectrum could appear distorted according to the neutrino oscillation hypothesis. The shapeonly analysis of the Nucifer data will be the first unambiguous test of the existence of a sterile neutrino, although the complete area of the reactor anomaly contour can not be covered by this experiment alone.

\subsection{Stereo}

The Stereo setup [6], based on experience from the Double Chooz and Nucifer experiments, is illustrated in figure 3. The target liquid scintillator, doped with $\mathrm{Gd}$, is contained in a $8 \mathrm{~mm}$ thick acrylic vessel. The section of the target is about $1 \mathrm{~m} \times 1 \mathrm{~m}$ and the length is $2 \mathrm{~m}$. The target vessel is immersed in another LS, not doped with Gd, contained in a second acrylic vessel. This $15 \mathrm{~cm}$ thick outer layer collects part of the energy of $\gamma$-rays escaping from the target, reducing the low energy tail of the detector response. 64 PMTs of 8 inches diameter are distributed across the lateral surfaces of the outer acrylic box. The roof and bottom planes are covered with diffusive white Teflon. Fixed optical coupling of the photomultipliers with thick 
lateral acrylic walls can be used to provide mechanical support and serve as a buffer layer for a more uniform detector response.

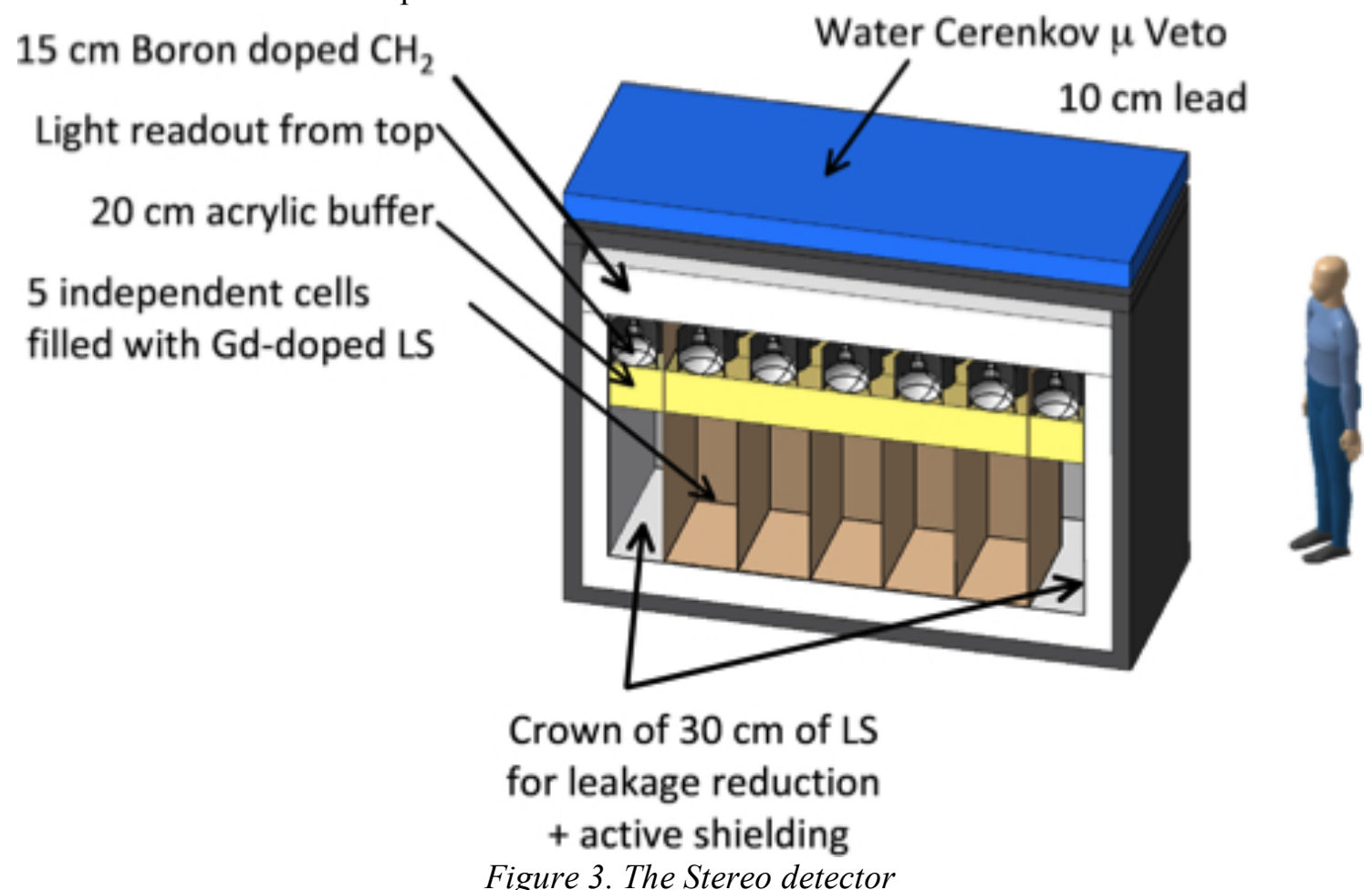

The Stereo detector will be installed in 2014 at the ILL reactor in Grenoble. Along the axis pointing toward the core, the detector is built long enough to see the phase of the new oscillation in the energy spectrum changing along this axis. To prove the existence of a sterile neutrino, emphasis is put on the shape-only analysis, looking for the relative deformation of the spectrum along the detector with as little as possible normalization input.

\subsection{Danss}

The DANSS detector will consist of highly segmented plastic scintillator with a total volume of $1 \mathrm{~m}^{3}$, surrounded with a composite shield of copper $(\mathrm{Cu})$, lead $(\mathrm{Pb})$ and borated polyethylene ( $\mathrm{CHB})$, and vetoed against cosmic muons with a number of external scintillator plates.

The basic element of DANSS is a polystyrene-based extruded scintillator strip $(1 \times 4 \times 100$ $\mathrm{cm}^{3}$ ) with a thin Gd-containing surface coating which is a light reflector and an (n, $\left.\gamma\right)$-converter simultaneously. The coating (about $0.1-0.2 \mathrm{~mm}$ ) is produced by co-extrusion and consists of polystyrene with $18 \%$ admixture of rutile and $6 \%$ of gadolinium oxide, so that the final $\mathrm{Gd}$ density is about $1.6 \mathrm{mg} / \mathrm{cm}^{2}$, which corresponds to $\approx 0.35 \% \mathrm{wt}$. Light collection from the strip is done via three wavelength-shifting Kuraray fibers $\mathrm{Y}-11, \oslash 1.2 \mathrm{~mm}$, glued into grooves along all the strip. An opposite (blind) end of each fiber is polished and covered with a mirror paint, which decreases a total lengthwise attenuation of a light signal down to $\approx 5 \% / \mathrm{m}$.

Each 50 parallel strips are combined into a module, so that the whole detector ( 2500 strips) is a structure of 50 intercrossing modules. Each 2 module is viewed by a compact photomultiplier tube (Hamamatsu R7600U-200) coupled to all 50 strips of the module via 100 
WLS fibers, two per strip. In addition, to get more precise energy and space pattern of an event, each strip is equipped with an individual multipixel photosensor (SiPM) operating in the Geiger mode and coupled to the strip via the third WLS fiber.

The detector is installed under the industrial $3 \mathrm{GWth}$ reactor of the Kalinin Nuclear Power Plant - KNPP) and a pilot version DANSSino is operated already [8]

\section{Experimental projects with sources}

The definitive test of the reactor antineutrino anomaly is the observation of the oscillation pattern as a function of $\mathrm{L} / \mathrm{E}$. This can be realized either by using a monochromatic neutrino source like ${ }^{51} \mathrm{Cr}$ or ${ }^{37} \mathrm{Ar}$ or by using an antineutrino emitter with a continuous beta spectrum. In the first case, with a liquid scintillator detector, the reaction is through the elastic scattering of neutrino on electrons, in the second case the antineutrino interact with the free protons through the inverse beta decay reaction.

\section{$4.1^{51} \mathrm{Cr}$ neutrino source}

Intense ${ }^{51} \mathrm{Cr}$ neutrino sources have been made in the past by irradiation of enriched chromium which is still available. ${ }^{51} \mathrm{Cr}$ emits two monoenergetic lines of neutrinos $(751 \mathrm{keV}$ and $480 \mathrm{keV}$ ) well suited to test the possible new oscillation at few meters.

\subsubsection{Dual metallic Gallium target experiment}

To investigate the sterile neutrino explanation for the Ga source experiments, an improved version of these measurements made by Sage will be used [9]. The plan is to place a ${ }^{51} \mathrm{Cr}$ source with initial activity of $3 \mathrm{MCi}$ at the center of a 50-tonne target of liquid $\mathrm{Ga}$ metal that is divided into two concentric spherical zones, an inner 8-tonne zone and an outer 42-tonne zone. If oscillations to sterile neutrinos do not occur, then at the beginning of irradiation there is a mean of 65 atoms of ${ }^{71} \mathrm{Ge}$ produced by the source per day in each zone. After an exposure period of a few days, the $\mathrm{Ga}$ in each zone is transferred to reaction vessels and the ${ }^{71} \mathrm{Ge}$ atoms produced by neutrino capture are extracted. These steps are the same as were used in the prior source experiments and are well tested. Finally, the Ge atoms are placed in proportional counters and their number is determined by counting the Auger electrons released in the transition back to ${ }^{71} \mathrm{Ge}$, which occurs with a half life of 11.4 days. A series of exposures is made, each of a few days duration, with the ${ }^{71} \mathrm{Ge}$ atoms from each zone measured in individual counters.

If oscillations to a sterile neutrino are occurring with mass-squared difference of $\Delta \mathrm{m}^{2}$ and mixing parameter $\sin ^{2}(2 \theta)$, then the rates in the outer and inner zones of gallium will be different.

\subsubsection{SoX phase $1[10]$}

The solar neutrino detector Borexino is perfectly suited to host a source experiment able to shed light on experimental hints, pointing to the possible existence of a sterile neutrino at the few $\mathrm{eV}^{2}$ mass scale. The extreme radiopurity achieved in the liquid scintillator acting as detection medium, make Borexino an ideal choice for the sterile neutrino experimental investigation. Preliminary studies indicate, in particular, that Borexino could be a well suited 
location both for an external neutrino source experiment (with an ultimate background of about $50 \mathrm{eV} /$ day mainly due to the irreducible contribution of solar neutrinos).

Neutrino source experiments have been part of the Borexino program since the very beginning of the project and thus a small tunnel was built right below the water tank, providing a location as close as $8.25 \mathrm{~m}$ to the scintillator inner vessel center. This tunnel is ready to be used. This location, together with other possible locations inside the detector which have been also investigated, is very appropriate for a sterile neutrino search with the current Borexino setup. The distance between the source and the detector, and the size of the detector itself, are of the order of few meters, thus comparable with the expected oscillation length : therefore a possible striking signature of the occurrence of the oscillation involving the putative sterile neutrino would be the development across the detector of a clearly visible "spatial wave of detected neutrino events.

\section{$4.2{ }^{144} \mathrm{Ce}-{ }^{144} \mathrm{Pr}$ antineutrino sources}

The elastic scattering of neutrino can be easely mimic by a Compton scattering, thus the constraints for an experiment with a neutrino source are severe. On the contrary the use of an antineutrino source allow the detection via IBD with a delayed coincidence. This offer an efficient rejection of gamma induced background.

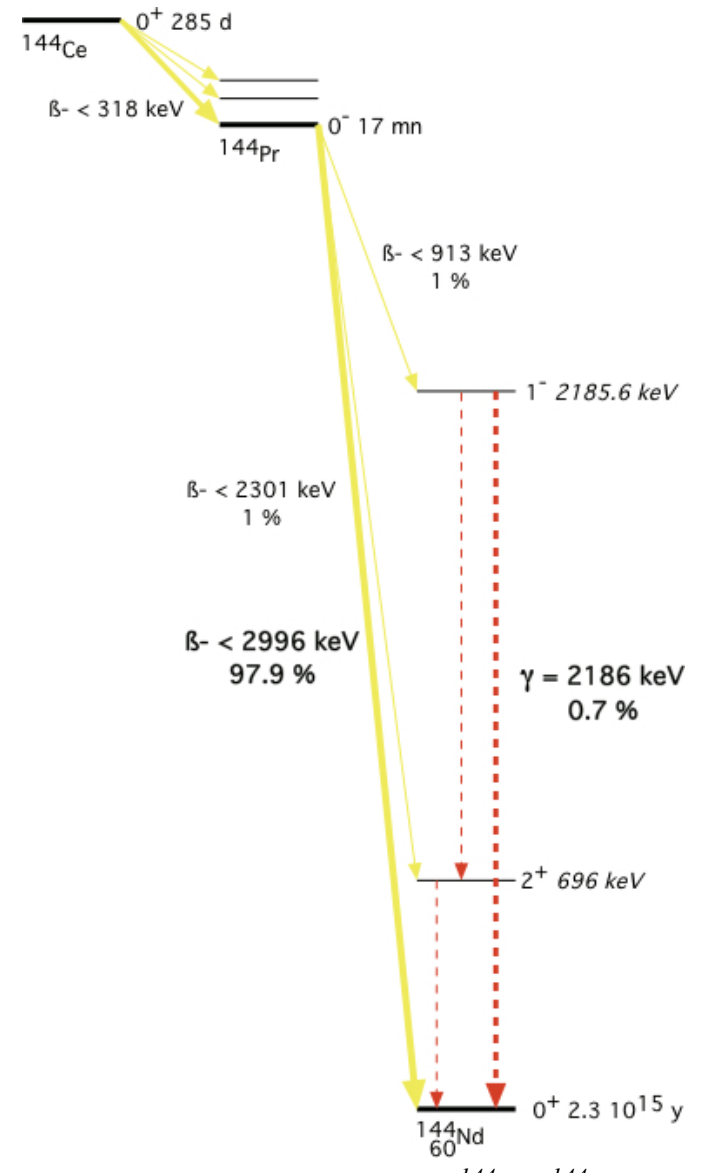

Fig.4. Decay scheme of ${ }^{144} \mathrm{Ce}_{-}{ }^{144} \mathrm{Pr}$
A suitable $\bar{v}_{e}$ source must have $\mathrm{Q}_{\beta}>1.8$ $\mathrm{MeV}$ (the IBD threshold) and a lifetime that is long enough $(\approx 1$ year) to allow for production and transportation to the detector. For individual nuclei, these two requirements are contradictory so we expect candidate sources to involve a long-lived low-Q nucleus that decays to a shortlived high-Q nucleus. Four such pairs ${ }^{144} \mathrm{Ce}^{144} \mathrm{Pr}$ $\left[\mathrm{Q}_{\beta}{ }^{144} \mathrm{Pr}=2.996 \mathrm{MeV}\right],{ }^{106} \mathrm{Ru}-{ }^{106} \mathrm{Rh},{ }^{90} \mathrm{Sr}^{9}{ }^{90} \mathrm{Y}$ and ${ }^{42} \mathrm{Ar}^{42} \mathrm{~K}$ were identified $[11,12]$. The first three are common fission products from nuclear reactors that can be extracted from spent fuel rods. While not minimizing the difficulty of doing this, the nuclear industry does have the technology to produce sources of the appropriate intensity, at the ppm purity level. Delays obtaining authorizations for transportation and deployment of the source into an underground laboratory should be addressed at the start of the project. Now we concentrate on the ${ }^{144} \mathrm{Ce}$ source (Fig. 4) because its high $\mathrm{Q}_{\beta}$ and because it is easier to extract chemically than ${ }^{106} \mathrm{Ru}$. 
We note also that it has a very low production rate of high energy $\gamma$-rays $(>1 \mathrm{MeV})$ from which the $\bar{v}_{e}$ detector must be shielded to limit background events. Finally, Cerium is present in fission products of uranium and plutonium at the level of a few percent. The specific activity of ${ }^{144} \mathrm{Ce}$ is $3.18 \mathrm{kCi} / \mathrm{g}$, but this radioactive isotope remains mixed with the other stable Cerium iosotopes. The heat output $7.90 \mathrm{~W} / \mathrm{kCi}$ allow the measurement of the activity at the $1 \%$ level with calorimetry.

\subsection{Detectors}

\subsubsection{KamLAND}

The CeLAND experiment aims at deploying a $75-100 \mathrm{kCi}$ of ${ }^{144} \mathrm{Ce}-{ }^{144} \mathrm{Pr}$ in the outer veto of the KamLAND detector. The source will be produced at Mayak in Russia, transported to the experimental site. The data taking will last 18 months. Thanks to the well known performances of the detector a virtually background free experiment could be envisaged. Both energy and interaction point can be known event by event and the L/E distribution could be plotted (fig. 5).

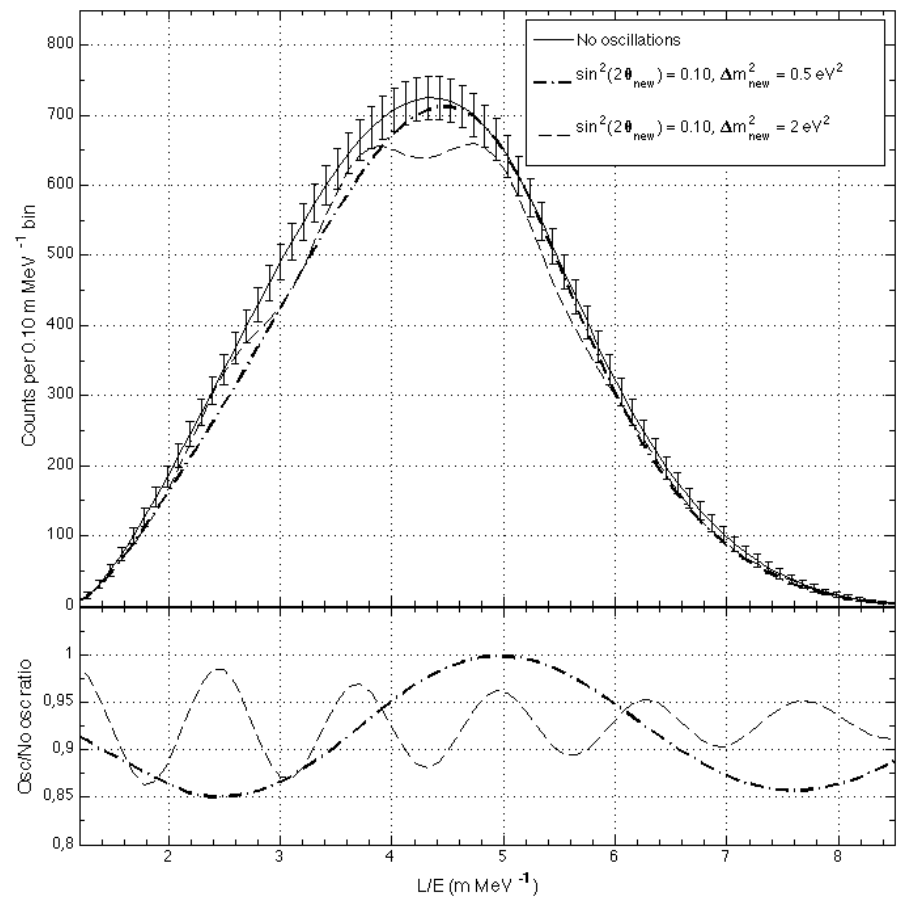

Figure 5. Expected result of CeLAND in $L / E$

\subsubsection{SoX phase 2 in Borexino}

A very similar program is considered in Borexino (SoX phase 2) after the test with the ${ }^{51} \mathrm{Cr}$ neutrino source. It is discribed in these proceedings.

\section{Conclusion}

In summary, there are a number of experimental results that appear anomalous in the context of the standard 3 neutrino framework, and can be explained by a sterile neutrino with mass around $1 \mathrm{eV}$. The need thus arises to provide a more ardent and complete test of 
the sterile neutrino hypothesis, which will unambiguously confirm or refute the interpretation of past experimental results. As shown in figure 6 future experiments will test the sterile neutrino hypothesis covering the full range of oscillation parameters with very different techniques.

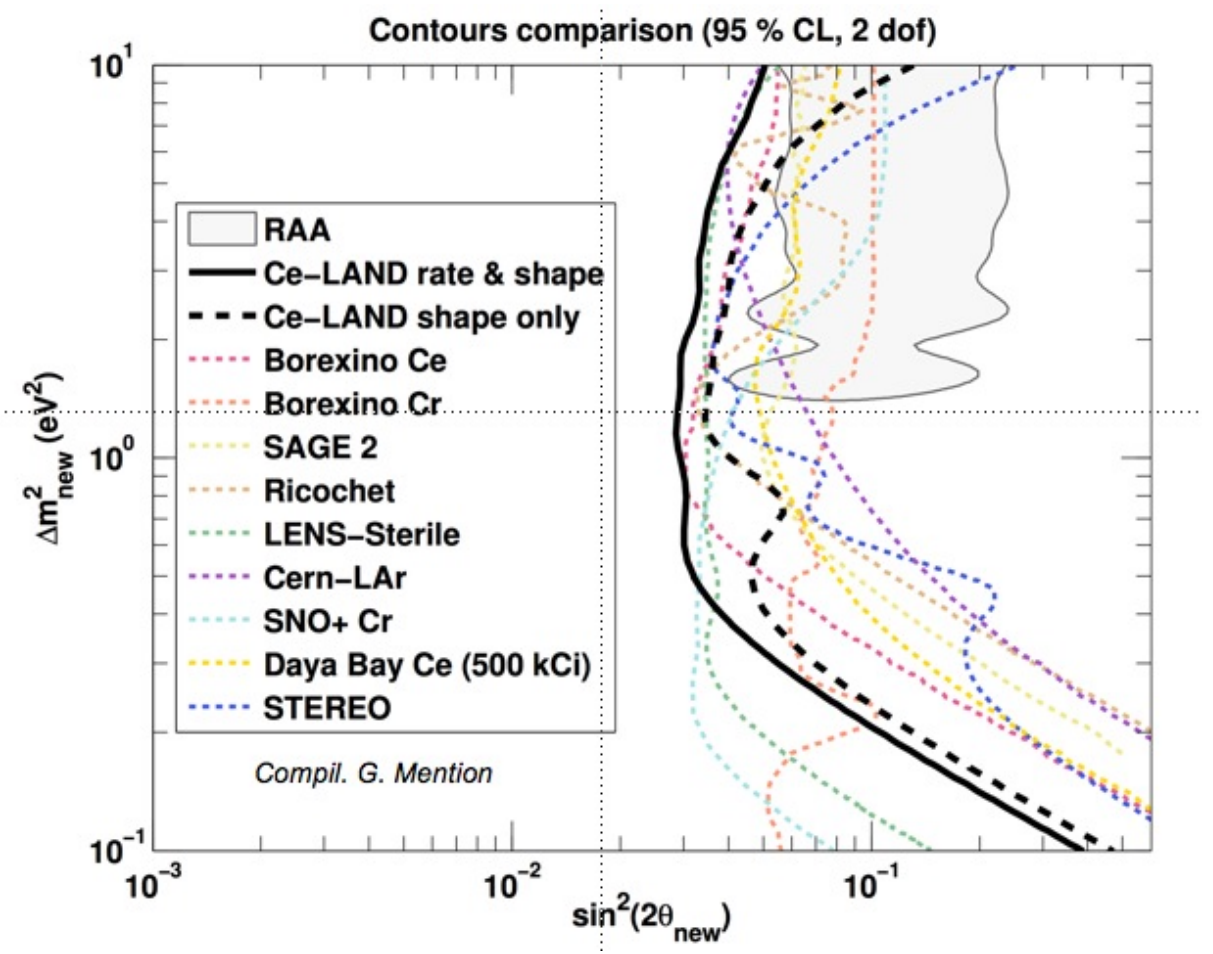

Figure 6. Comparison of the different experiments testing the sterile neutrino hypothesis

\section{References}

[1] Th. A. Mueller et al., Phys. Rev. C83 (2011) 054615

[2] G. Mention et al., Phys. Rev. D83, 073006 (2011).

[3] F. Von Feilitzsch, A. Hahn, and K. Schreckenbach, Phys.Lett. B118, 162 (1982)

[4] P. Huber, Determination of antineutrino spectra from nuclear reactors, Physical Review C 84 (2) (2011) 024617.

[5] A. Hoummada et al., Appl. Radiat. Isot. 46 (1995) 449

[6] K. N. Abazajian, et al., Light Sterile Neutrinos: A White Paper, arXiv :1204.5376.

[7] A. Porta et al., IEEE Transactions on Nuclear Science 57, 2732 (2010).

[8] I. Alekseev et al., DANSSino: a pilot version of the DANSS neutrino detector, arXiv :1305.3350.

[9] V.N. Gavrin et al., Gallium experiments with artificial neutrino sources as a tool for investigation of transition to sterile states, arXiv :1006.2103 (2010).

[10] M. Pallavicini, SoX, in these proceedings

[11] M. Cribier et al., Proposed Search for a Fourth Neutrino with a PBq Antineutrino Source, Phys. Rev. Lett. 107, 201801 (2011).

[12] V.N. Kornoukhov, ITEP report No. 90, Moscow, 1994. 